\title{
Glaucome primitif à angle ouvert précoce
}

\author{
Austin R. Lifferth, OD, FAAO \\ Clinique externe \\ pour anciens combattants \\ The Villages, Floride, \\ États-Unis
}

\section{Introduction}

Le glaucome est la principale cause de cécité irréversible dans le monde et devrait toucher plus de 79,6 millions de personnes d'ici 2020, dont plus de $10 \%$ seront atteintes de cécité bilatérale. ${ }^{1}$

Cette neuropathie optique progressive multifactorielle provoque des lésions caractéristiques à la couche de fibres nerveuses rétiniennes qui finiront par entraîner des lacunes glaucomateuses du champ visuel s'ils ne sont pas traités. Malheureusement, ces lacunes du champ visuel sont difficiles à détecter pour le patient avant les stades plus avancés et, par conséquent, le glaucome précoce est habituellement asymptomatique. ${ }^{2}$

Cet article présente un cas qui concorde avec les études des populations selon lesquelles jusqu'à la moitié des personnes atteintes de glaucome ne savent pas qu'elles ont contracté la maladie. ${ }^{3}$

\section{ÉTUDE DU CAS}

Un homme de 71 ans de type caucasien en bonne santé s'est présenté à notre bureau comme un nouveau patient qui se plaignait d'une vision légèrement floue, O.D. $=$ O.S., à distance et de près.

Le patient a déclaré qu'il avait réalisé un examen de la vue « 6 à 7 ans » avant notre examen et qu'on lui avait dit qu'il avait des « symptômes de glaucome ». Toutefois, il n'a pas reçu de diagnostic de glaucome et n'a pas reçu de traitement supplémentaire. Il a nié tout antécédent familial de glaucome, était en bonne santé générale et n’a signalé aucune autre difficulté mettant en cause l'un ou l'autre des yeux.

Le patient s'automédicamentait avec de l'aspirine de $81 \mathrm{mg}$, des multivitamines, des capsules d'huile de poisson, un complexe de vitamine B et des gélules de levure de riz rouge, tous pris une fois par jour.

Sa tension artérielle la plus récente était de $150 / 67$ à 14 h 5 en position assise, son taux d'AlC était de 5,8 \% et son taux de glycémie était de $116 \mathrm{mg} /$ $\mathrm{dL}$ à $14 \mathrm{~h} 37$. Son indice de masse corporelle était de 28 . Aucune allergie aux médicaments n'a été signalée.

Les acuités visuelles à distance et au trou sténopéïque naturelles du patient étaient les suivantes :

O.D. : 20/60-1 Sténopéïque : 20/25

O.S. : 20/40+2 Sténopéïque : 20/25+2

La réfraction subjective et l'acuité visuelle la mieux corrigée étaient les suivantes :

O.D. : $-0,75-1,50 \times 07020 / 20$

O.S. : $-0,25-1,0 \times 06220 / 25+1$ 
L’examen avec la lampe à fentes a révélé une légère blépharite bilatérale, une conjonctive claire et une cornée claire sans pigment endothélial ni précipités kératiques. La chambre antérieure était profonde et sans inflammation selon la technique d'estimation de l'angle Van Herick, et l'iris était normal sans signes d'atrophie, de synéchie postérieure évidente ou de défaut de transéclairage. Les pressions intraoculaires (PIO) étaient de 17 O.D. et de 15 O.S. à 8 h 1 par tonométrie par aplanation de Goldmann.

Un examen de dilatation a révélé des traces de sclérose nucléaire et d'opacités corticales sans preuve de pseudoexfoliation ou de pigment. La macula, les vaisseaux et la périphérie étaient tous normaux, O.D. et O.S. Il y avait un décollement du vitré postérieur O.D. et O.S. sans signe d'anomalie rétinienne périphérique.

Le disque du nerf optique était de taille moyenne à grande O.D.>O.S. en se basant sur la hauteur du disque vertical à l'aide du graticule de la lampe à fente ajusté et d'une lentille Volk $78 \mathrm{D}$ ayant un facteur de correction de $1,2 \mathrm{x}$. La cupule optique était d'une profondeur modérée, avec des points laminaires visibles précoces O.U. Il y avait une légère atrophie parapapillaire de zone alpha, mais aucun signe de pâleur ni d'hémorragie du disque O.U. Il y avait un très léger défaut cunéiforme de la couche de fibre nerveuse rétinienne inférieure associé à un amincissement de l'anneau neurorétinien inférieur, à une mise à vif des vaisseaux inférieurs et à un rétrécissement de l'artériole inférieure, O.U. De plus, l'anneau supérieur était suspect pour la neuropathie optique glaucomateuse O.U., et il y avait des signes de mise à vif précoce des vaisseaux O.D.>O.S. et d'amincissement relatif par rapport à d'autres secteurs du nerf optique. On a estimé que les rapports cupule-papille étaient de 0,7 v/0,7 h O.D. et de 0,75 v/0,7 h O.S.

Des photos de référence, une tomographie par cohérence optique (TCO) et une analyse du disque du nerf optique (DNO) et de la couche de fibres nerveuses rétiniennes (CFNR) ont été obtenues. L'imagerie subjective et objective a confirmé les résultats de l'examen clinique, comme le montrent les figures 1 et 2.

Figure 1 : Images rétiniennes montrant des défauts de la couche de fibres nerveuses rétiniennes localisées dans la zone temporale inférieure et l'amincissement de l'anneau neurorétinien temporal inférieur associé. Remarquez l'amincissement de l'anneau neurorétinien supérieur précoce relatif O.U.

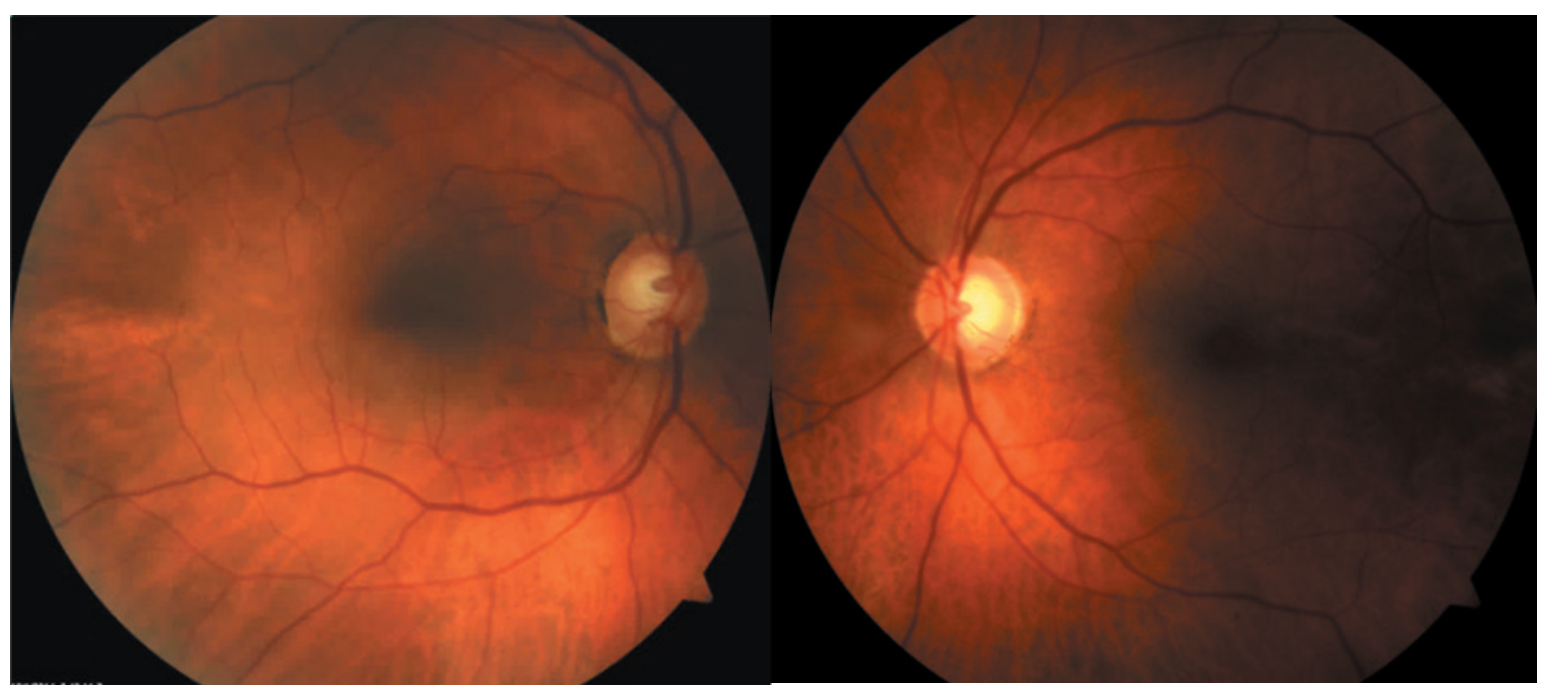

Le patient a reçu un diagnostic provisoire de glaucome précoce, O.S.>O.D., et il a été invité à revenir dans le mois pour des mesures de PIO répétées avec gonioscopie de référence, pachymétrie et examen du champ visuel de seuil.

Au cours du rendez-vous de suivi d'un mois, nous avons constaté que le patient avait une acuité stable et ne se plaignait pas davantage de ses yeux. Ses pressions intraoculaires étaient légèrement plus élevées que le niveau de référence, à 21 O.D., 19 O.S. à 10 h 42 par tonométrie par aplanation de Goldmann. La pachymétrie a donné des mesures d'épaisseur de la cornée centrale légèrement plus minces que la moyenne, à 524 u O.D., 525 u O.S. La gonioscopie a montré que le corps ciliaire était visible dans les quatre quadrants avec un iris plateau, une pigmentation légère du trabéculum cornéoscléral et aucune trace de synéchie antérieure périphérique ou de récession d’angle O.D., O.S. 
Figure 2 : Tomographie par cohérence optique (TCO) et analyse du disque du nerfoptique (DNO) et de la couche de fibres nerveuses rétiniennes (CFNR) O.U. Remarquez les décollements du vitré postérieurs nasaux bilatéraux avec les artéfacts associés observés sur les cartes d'épaisseur et de déviation de la CFNR. De plus, l'amincissement de la CFNR est localisé et, à ce titre, n'est indiqué que sur la carte en forme d'horloge de la CFNR, tout en apparaissant comme «normal » sur la carte des quadrants de la CFNR.

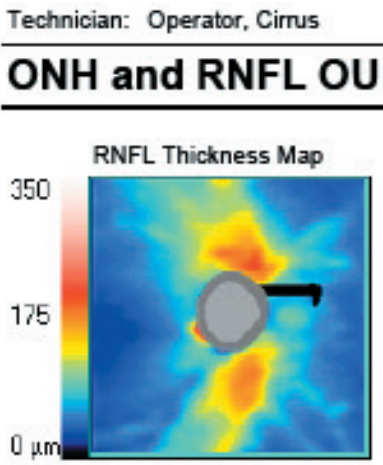

RNFL Deviation Map

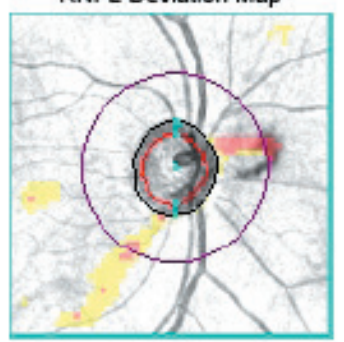

Disc Center $(0.06,0.15) \mathrm{mm}$ Extracted Horizontal Tomogram

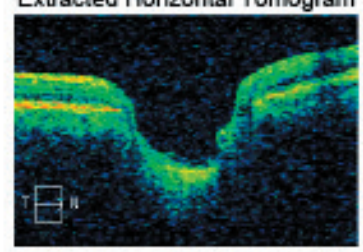

Extracted Vertical Tomogram

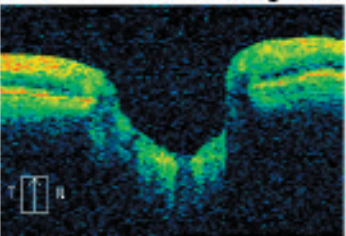

RNFL Circular Tomogram

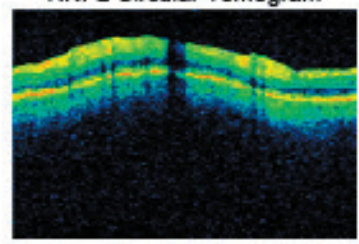

Signal Strength: $8 / 10$

$8 / 10$

\begin{tabular}{|r|c|c|}
\hline \multicolumn{1}{|c|}{ A } & OD & OS \\
\hline Arerage RNFL Thizkness & $97 \mu \mathrm{m}$ & $96 \mu \mathrm{m}$ \\
\hline FNFL Symmetry & \multicolumn{2}{|c|}{$90 \%$} \\
\hline Rin Ares & $0.00 \mathrm{~mm}^{2}$ & $0.05 \mathrm{~mm}^{2}$ \\
\hline Disc Ares & $2.14 \mathrm{~mm}^{2}$ & $1.88 \mathrm{~mm}^{2}$ \\
\hline Average C.D Ratio & 0.74 & 0.71 \\
\hline Vatica C.D Ratio & 0.79 & 0.78 \\
\hline Cup Voure & $0.588 \mathrm{~mm}^{2}$ & $0.412 \mathrm{~mm}^{2}$ \\
\hline
\end{tabular}

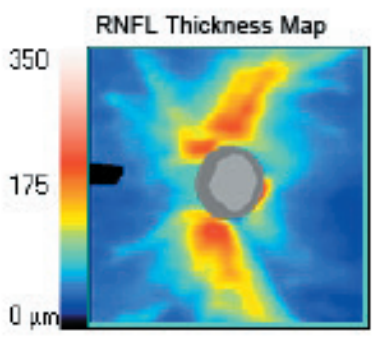

RNFL Deviation Map

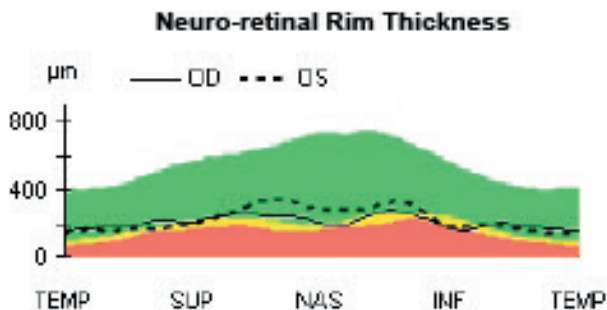

RNFL Thickness
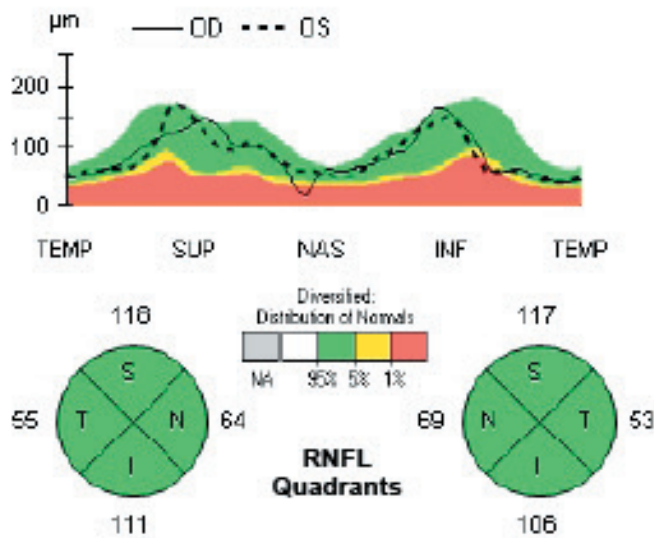

111

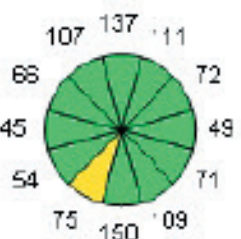

106

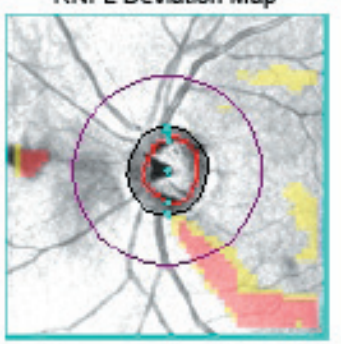

Disc Center(0.03,0.12) $\mathrm{mm}$

Extracted Horizontal Tomogram

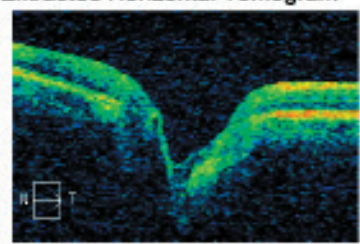

Extracted Vertical Tomogram

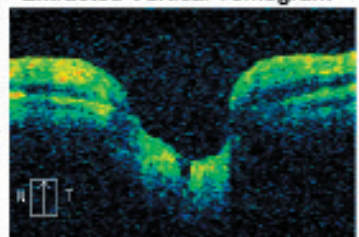

RNFL Circular Tomogram

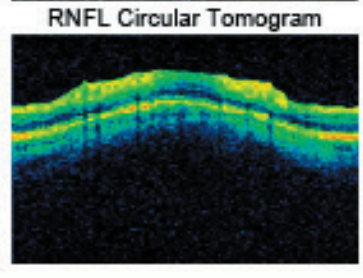

Le seuil de référence pour l'examen du champ visuel (Humphrey 24-2 SITA Fast) a montré un O.D. de sensibilité normale avec une bonne fiabilité ( $0 / 10$ pertes de fixation, $5 \%$ de faux positifs et $0 \%$ de faux négatifs) et plusieurs défauts nasaux supérieurs à enfoncement focal pour l'O.S. avec une moins bonne fiabilité (5/11 pertes de fixation, $3 \%$ de faux positifs et $8 \%$ de faux négatifs). 
Figure 3: Les tracés de référence de champ visuel du test Humphrey 24-2 SITA Fast ne montrent que les défauts à enfoncement focal pour l'O.S. uniquement.
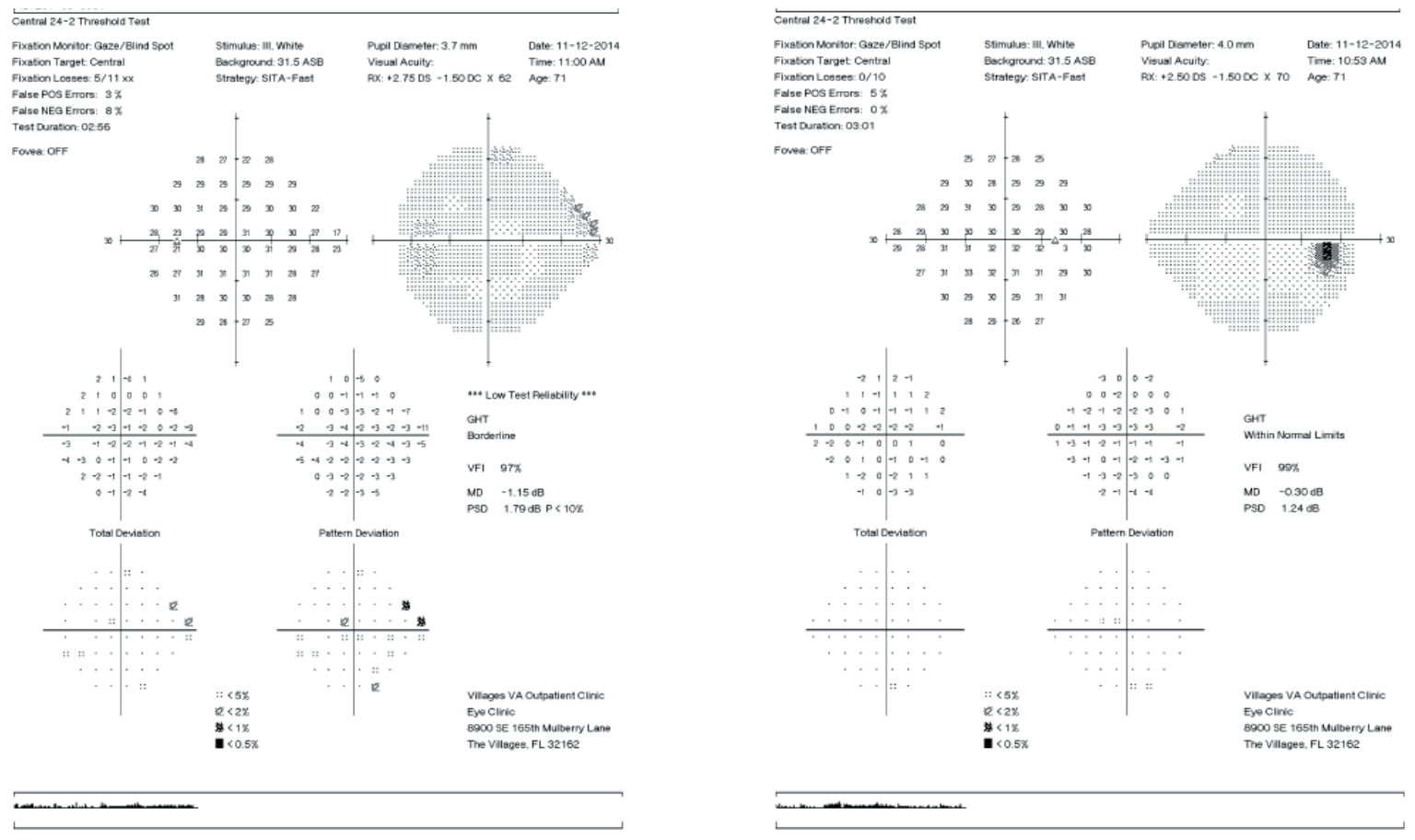

En raison de l'apparence gonioscopique normale de l'angle, le patient a reçu un diagnostic plus précis de glaucome primitif à angle ouvert précoce O.S.>O.D. avec des défauts périmétriques précoces discutables de l'O.S. basés sur l'algorithme du test SITA Fast. Bengtsson et Heijl ${ }^{4}$ ont constaté que les temps d'examen du SITA Fast (5,0 min en moyenne) étaient beaucoup plus courts que ceux de seuil complet (14,6 min en moyenne) et du Fastpac (9,4 min en moyenne), mais qu'ils étaient relativement égaux en termes de reproductibilité. Toutefois, comme on pouvait s'y attendre, la sensibilité à la détection des défauts peu profonds (subtils) était plus grande lors des examens de seuil complet. En d'autres termes, les défauts peu profonds relevés lors des examens de seuil complet étaient de moins en moins visibles (peut-être même absents) avec les examens Fastpac et SITA Fast, tandis que la détection de défauts focaux plus profonds était relativement identique avec les trois stratégies.

En collaborant avec le patient, nous avons décidé de surveiller de près la maladie à ce moment-là, sans traitement, jusqu'à ce que des examens structuraux et fonctionnels répétés confirment les défauts soupçonnés (ou la progression suggérée) et dans le but d'établir les pressions intraoculaires de référence à divers points temporels diurnes. Cependant, d'après les conclusions sur ce cas jusqu'à maintenant et d'après les études Early Manifest Glaucoma Trial (EMGT) et Collaborative Normal Tension Glaucoma Study (CNTGS), le traitement sera probablement commencé pour contribuer à réduire le risque de progression de la maladie. De plus, et peut-être plus pertinent pour notre patient, Kim et coll. ont constaté qu'une réduction suffisante de la PIO ralentissait la progression de la maladie même chez les patients chez qui l'on suspecte un glaucome pré-périmétrique. ${ }^{5}$

On a demandé au patient de revenir dans notre bureau 3 ou 4 mois plus tard pour répéter les tests de tonométrie, de pachymétrie et de champ visuel de seuil 24-2, ainsi que pour une analyse des cellules ganglionnaires par tomographie par cohérence optique de référence et un examen du champ visuel 10-2. Pour résumer, l'importance (et les avantages) de l'analyse des dix degrés centraux du champ visuel ne peut être surévaluée : environ la moitié des yeux atteints de glaucome léger à modéré présentaient des défauts dans les trois degrés centraux, ${ }^{6}$ onze yeux avec des champs visuels de seuil 24-2 normaux à l'extérieur des dix degrés centraux présentaient des défauts dans la région de l'arc dans les dix degrés centraux avec un examen 10-2, neuf pour cent des champs visuels de seuil 30-2 normaux 
des patients chez qui l'on suspecte un glaucome ou qui présentent un glaucome précoce étaient en fait catégorisés comme anormaux avec un examen 10-2 ${ }^{8}$ et près de la moitié des yeux présentent des lésions glaucomateuses maculaires lors de l'examen 10-2 alors qu'ils sont considérés comme normaux avec l'examen 24-2.9

\section{DISCUSSION}

Weinreb et coll. proposent un continuum du glaucome : un spectre des étapes structurelles et fonctionnelles du glaucome dans lequel le patient progresse généralement d'un état « normal » et d'une maladie asymptomatique vers une cécité fonctionnelle, les changements glaucomateux structurels précédant généralement les symptômes fonctionnels. ${ }^{10}$ L'Association mondiale du glaucome décrit également cette relation temporelle entre les changements structuraux et fonctionnels tout du long de la maladie. ${ }^{11}$ Les deux représentations suggèrent que les changements structurels sont habituellement détectés avant les changements fonctionnels. Cependant, et à titre de rappel important provenant de l'étude rétrospective d'Alasil et coll. et de leurs constatations d'un « point de basculement » structurel et fonctionnel, des tests structuraux et fonctionnels sont toujours nécessaires pour évaluer les lésions glaucomateuses précoces. ${ }^{12}$

Les Preferred Practice Pattern (pratiques à privilégier) de l'American Academy of Ophthalmology (AAO PPP) pour le glaucome primitif à angle ouvert indiquent que le glaucome léger (précoce) se caractérise par des « anormalités du nerf optique cohérentes avec le glaucome comme un amincissement diffus, un rétrécissement focal ou l'excavation de l'anneau neurorétinien de la papille, surtout aux pôles inférieur ou supérieur, un rétrécissement progressif de l'anneau neurorétinien avec une augmentation associée de la cupule de la papille, des anormalités diffuses ou localisées de la CFNR (couche des fibres nerveuses rétiniennes) parapapillaire, surtout aux pôles inférieur ou supérieur, des hémorragies de l'anneau neurorétinien, de la CFNR parapapillaire ou de la lame criblée, ou une asymétrie de l'anneau neurorétinien de la papille entre les deux yeux, cohérente avec la perte de tissus neuronaux » en présence d'un « champ visuel normal examiné par périmétrie automatique standard. $»^{13}$

Dans les lignes directrices de pratique clinique de l'American Optometric Association (AOA CPG), le glaucome léger est défini comme un nerf optique avec un « rétrécissement concentrique léger ou un rétrécissement localisé partiel de l'anneau neurorétinien; une hémorragie de la papille, (ou) une asymétrie cupule/papille ». De plus, la couche de fibres nerveuses montre un « réflexe photomoteur moins important, des stries fines à la texture, (ou) de grands vaisseaux sanguins rétiniens qui semblent relativement clairs tandis que les vaisseaux sanguins rétiniens moyens sont moins flous et les petits vaisseaux sanguins rétiniens sont flous ». Cependant, contrairement aux AAO PPP, les AOA CPG mentionnent que le glaucome précoce peut présenter des « scotomes paracentraux isolés, des défauts dans la région de l'arc ou nasale et que les lésions sont limitées à un hémichamp avec moins de $25 \%$ des points impliqués et avec une déviation moyenne inférieure à $-6 \mathrm{~dB}$. $»^{14}$ Une définition plus succincte qui semble relier les deux définitions précédentes est celle donnée par Song et Caprioli : « une neuropathie optique progressive définie par des changements structuraux caractéristiques du nerf optique et des changements fonctionnels correspondants du champ visuel $\gg .^{15}$

Néanmoins, lorsque la perte fonctionnelle est détectable, la gravité de la neuropathie optique glaucomateuse augmente avec celle de la perte du champ visuel, comme le montrent $\mathrm{Ng}$ et coll. ${ }^{16}$

Le patient en question présentait plusieurs facteurs de risque pour le glaucome à angle ouvert, ${ }^{1718}$ comme sa pression intraoculaire légèrement élevée associée à une faible fluctuation ${ }^{19}$ (bien que cela ne soit basé que sur deux prises de mesure isolées) et son âge légèrement avancé. ${ }^{20,21}$ Toutefois, d'autres facteurs de risque qui ne s'appliquaient pas à ce cas, mais qui doivent aussi être pris en compte, comprennent la présence d'exfoliation lenticulaire, ${ }^{22,23}$ des hémorragies glaucomateuses de la papille, ${ }^{24-28}$ une ascendance afro-américaine ${ }^{29}$ des antécédents de glaucome au premier degré ${ }^{30}$ et des antécédents généraux de diabète ${ }^{31}$ ou d'hypertension..$^{32-34}$

Le principal signe clinique qui a été le plus convaincant pour ce patient était l'apparence du nerf optique et de la couche de fibres nerveuses rétiniennes. Plus précisément, les défauts de la couche de fibres nerveuses rétiniennes inférieures associés à l'amincissement de l'anneau neurorétinien, à la mise à vif des vaisseaux inférieurs et au rétrécissement de l'artériole inférieure (et le possible amincissement de l'anneau supérieur) sont tous caractéristiques du glaucome précoce. En raison de ce modèle à privilégier de perte de l'anneau neurorétinien, la règle mnémonique ISNT $^{35,36}$ s'est avérée très efficace pour différencier les nerfs optiques normaux de ceux qui ont des lésions glaucomateuses précoces. De plus, l'absence de pâleur de l'anneau contribue à éliminer d'autres neuropathies optiques (ischémique, par infiltration, traumatique, toxique, métabolique et compressive) qui pourraient également provoquer 
des lésions à la couche de fibres nerveuses rétiniennes et un rétrécissement de l'artériole, et qui nécessiteraient des examens systémiques plus poussés, comme des analyses sanguines et la neuroimagerie. ${ }^{37-39}$ Des photos de référence ont été prises afin de contribuer à la surveillance de la progression structurelle ${ }^{40}$ à l'avenir qui pourrait se manifester par un élargissement des lésions à la couche de fibres nerveuses (emplacements de la progression future et corrélation avec les lésions du champ visuel ${ }^{41}$ ), une augmentation de l'épaississement de l'anneau, de la mise à vif des vaisseaux ou du rétrécissement de l'artériole, de l'atrophie parapapillaire ou une plus forte nasalisation du tronc des vaisseaux rétiniens centraux.

L'évaluation subjective des photos en série et l'imagerie objective (TCO) sont des évaluations structurelles complémentaires utilisées de concert avec l'évaluation fonctionnelle (champ visuel) régulière pour surveiller la progression. Elles sont toutes nécessaires : l'Association mondiale du glaucome indique qu'« actuellement, aucun test en particulier ne peut être considéré comme la norme de référence parfaite pour la détection de la progression structurelle glaucomateuse ou fonctionnelle. $»^{41}$ Appuyant cette position, Banegas et coll. indiquent que dans leur étude observationnelle portant sur 246 yeux, la progression glaucomateuse était détectée pour $6,9 \%$ des yeux par des photos stéréoscopiques, pour $15 \%$ par des examens du champ visuel et pour $25,6 \%$ par un logiciel d'analyse de la progression guidé par TCO. Il est intéressant de remarquer que tous les cas ayant démontré une progression ont été découverts par des photos stéréoscopiques, la périmétrie ou la TCO uniquement. Cela suggère qu'il existe un faible pourcentage d'accord positif parmi les méthodes d'évaluation et souligne l'importance de les utiliser toutes trois pour surveiller les changements. ${ }^{42,43}$ Dans ce cas, l'apparence clinique du disque du nerf optique était très bien corrélée à l'analyse par TCO de la CFNR de référence, ce qui fournit des mesures de référence structurelles subjectives et objectives.

Appuyant le diagnostic de glaucome fondé uniquement sur l'apparence du nerf optique sans attendre le développement de lésions glaucomateuses du champ visuel corrélées, Sommer et coll. suggéraient en 1977 que les lésions de la couche de fibres nerveuses glaucomateuses (comme celles observées chez ce patient) peuvent se développer de nombreuses années avant qu'apparaissent des lésions glaucomateuses fiables du champ visuel. ${ }^{44}$ De plus, et plus récemment, Kuang et coll. ont démontré que les lésions de la CFNR observées lors de l'examen par TCO étaient détectables jusqu'à huit ans avant l'apparition des lésions glaucomateuses associées du champ visuel..$^{45}$ Allant dans le même sens que ces constatations, des études histologiques ont montré que jusqu'à $50 \%$ des cellules ganglionnaires rétiniennes sont perdues avant l'apparition de lésions du champ visuel cliniquement détectables ${ }^{46}$ dont découle un modèle de corrélation à «bâton brisé » entre l'épaisseur des fibres nerveuses rétiniennes et les champs visuels glaucomateux, comme précédemment décrit par Alasil et coll. ${ }^{12}$

Comme nous l'avons mentionné précédemment, malgré les renseignements corrélés sur le plan clinique suggérant un glaucome à angle ouvert précoce, la collaboration avec le patient a permis d'expliquer que nous n'avons pas entrepris de traitement pour les trois raisons suivantes:

1. Pour établir un intervalle de référence de la PIO à la lumière de la fluctuation potentielle des mesures initiales de PIO;

2. Pour établir la reproductibilité et la fiabilité du champ visuel de référence; ${ }^{47}$

3. Pour établir le taux de progression, en reconnaissant que ce ne sont pas tous les patients atteints de glaucome qui progresseront au point où les symptômes visuels affectent leurs activités quotidiennes. ${ }^{48}$

\section{CONCLUSION}

Une pression intraoculaire élevée est le principal facteur de risque (et actuellement le seul facilement modifiable) du développement d'un glaucome et de sa progression. ${ }^{49-53}$ Ainsi, si un traitement est nécessaire à l'avenir, nous travaillerons avec le patient pour déterminer un intervalle de PIO cible personnalisé et unique, la « limite supérieure d'un intervalle de PIO à laquelle on juge que d'autres lésions du nerf optique devraient être retardées $»,{ }^{54}$ et pour minimiser la perte de champ visuel associée. ${ }^{55} \mathrm{Il}$ est très important d'équilibrer cet intervalle dynamique de PIO avec les facteurs de qualité de vie, notamment l'estimation du risque à vie d'invalidité visuelle pour le patient, les effets secondaires possibles des traitements (topique, laser, chirurgie du glaucome la moins invasive possible), le fardeau financier du traitement et la technique ou la capacité d'instillation. 
Le glaucome primitif à angle ouvert peut ne pas être détecté à ses premiers stades en raison de sa nature asymptomatique, des changements morphologiques subtils du nerf optique et, souvent, de sa présentation pré-périmétrique. Pour ces raisons, nous devons être vigilants, car les chances de préserver la vision fonctionnelle du patient à vie sont les plus élevées si nous pouvons diagnostiquer le glaucome plus tôt et, au besoin, le traiter plus tôt. •

\section{RÉFÉRENCES}

1. Quigley, H., Broman, A. The number of people with glaucoma worldwide in 2010 and 2020. Br J Ophthalmol Mars 2006; 90(3):262-7.

2. Weinreb R., Aung T., Medeiros F. The pathophysiology and treatment of glaucoma: a review. JAMA 14 mai 2014; 311(18):1901-11.

3. Hennis A., Wu S., Nemesure B., Honkanen R., Leske M. Awareness of incident open-angle glaucoma in a population study: the Barbados Eye Studies. Ophthalmology, octobre 2007; 114(10):1816-21.

4. Bengtsson B., Heijl A.. SITA Fast, a new rapid perimetric threshold test. Description of methods and evaluation in patients with manifest and suspect. Acta Ophthalmol Scand 1998; 76:431-7.

5. Kim K.O., Jeoung J.W., Kim D.M., et coll. Long-term follow-up in preperimetric open-angle glaucoma: progression rates and associated factors. Am J Ophthalmol 2015 Jan; 159(1):160-8.el-2

6. Schiefer U., Papageorgiou E., Sample P.A., et coll. Spatial pattern of glaucomatous visual field loss obtained with regionally condensed stimulus arrangements. Invest Ophthalmol Vis Sci 2010; 51(11):5685-9.

7. Hood D.C., Raza A.S., de Moraes C.G., et coll. Initial arcuate defects within the central 10 degrees in glaucoma. Invest Ophthalmol Vis Sci 2011; 52(2):940-6.

8. Langerhorst C.T., Carenini L.L., Bakker D., De Bie-Raakman M.A.C. Measurements for description of very early glaucomatous field defects. Perimetry Update 1996/1997. New York (NY): Kugler Publications; 1997:67-73.

9. Grillo L., Wang D., Hood D., et coll. The 24-2 visual field test misses central macular damage confirmed by the 10-2 visual field test and optical coherence tomography. Transl Vis Sci Technol, 14 avril 2016; $5(2): 15$

10. Weinreb R.N., Friedman D.S., Fechtner R.D., et coll. Risk assessment in the management of patients with ocular hypertension. Am J Ophthalmol 2004 Sept.; 138(3):458-67.

11. Harwerth RS. Histopathology underlying glaucomatous damage - I in Glaucoma Diagnosis Structure and Function Consensus Series 1. La Haye, Pays-Bas; Kugler Publications: 2004:p.14.

12. Alasil T., Wang K., Yu F., Field M.G. Correlation of retinal nerve fiber layer thickness and visual fields in glaucoma: a broken stick model. Am J Ophthalmol 2014; 157 (5)953-9.

13. Preferred Practice Pattern Guidelines. Primary Open-Angle Glaucoma. American Academy of Ophthalmology; 2010.

14. American Optometric Association Clinical Practice Guidelines; 2010: 43 .

15. Song B.J., Caprioli J. Measuring glaucoma progression in clinical practice in Glaucoma - Medical Diagnosis and Therapy. Elsevier Limited 2015. Chapitre 23:268-76.

16. Ng M., Sample P.A., Pascual J.P. et coll. Comparison of visual field severity classification systems for glaucoma. J Glaucoma 2012; 21(8):551-61.

17. Chen P.P. Risk and risk factors for blindness from glaucoma. Curr Opin Ophthalmol 2004; 15(2):107-11.

18. Fechtner, R.D., Khouri, A.S. Evolving global risk assessment of ocular hypertension to glaucoma. Curr Opin Ophthalmol 2007; 18(2):104-9.

19. Wilensky J.T,. Gieser D.K., Dietsche M.L. et coll. Individual variability in the diurnal intraocular pressure curve. Ophthalmology 1993; 100(6):940-4.

20. Leske M.C., Connell A.M., Wu S.Y. et coll. Risk factors for openangle glaucoma. The Barbados Eye Study. Arch Ophthalmol 1995; 113(7):918-24.
21. Leske M.C., Wu S.Y., Hennis A et coll. Risk factors for incident open-angle glaucoma: the Barbados Eye Studies. Ophthalmology 2008; 115(1):85-93.

22. Jeng S.M., Karger R.A., Hodge D.O., Burke J.P., Johnson D.H., Good M.S. The risk of glaucoma in pseudoexfoliation syndrome. J Glaucoma 2007; 16(1):117-21.

23. Grødum, K., Heijl, A., Bengtsson, B. Risk of glaucoma in ocular hypertension with and without pseudoexfoliation. Ophthalmology 2005; $112(3): 386-90$.

24. Suh M.H., Park K.H. Pathogenesis and clinical implication of optic disk hemorrhage in glaucoma. Surv Ophthalmol 2014; 59:19-29.

25. Healey, P., Mitchell, P., Smith, W., Wang, J. Optic disc hemorrhages in a population with and without signs of glaucoma. Ophthalmology, février 1998; 105(2):216-23.

26. Siegner S.W., Netland P.A. Optic disc hemorrhages and progression of glaucoma. Ophthalmology 1996; 103:1014-24.

27. Uhler, T., Piltz-Seymour, J. Optic disc hemorrhages in glaucoma and ocular hypertension: implications and recommendations. Curr Opin Ophthalmol Mars 2008; 19(2):89-94

28. Suh, M., Park, K. Period prevalence and incidence of optic disc haemorrhage in normal tension glaucoma and primary open-angle glaucoma. Clin Exp Ophthalmol août 2011; 39(6):513-9.

29. Tielsch J.M., Sommer A., Katz J. et coll. Racial variations in the prevalence of primary open-angle glaucoma. The Baltimore Eye Survey. JAMA 1991; 266(3):369-74

30. Gordon M.O., Torri V., Miglior S. et coll. Validated prediction model for the development of primary open-angle glaucoma in individuals with ocular hypertension. Ophthalmology 2007; 114(1):10-19.

31. Zhou, M., Wang, W., Huang, W., Zhang, X. Diabetes mellitus as a risk factor for open-angle glaucoma: a systematic review and metaanalysis. PLoS One, 19 août 2014; 9(8):e102972.

32. Tielsch J.M., Katz J., Sommer A. et coll. Hypertension, perfusion pressure, and primary open-angle glaucoma. A population-based assessment. Arch Ophthalmol 1995; 113(2):216-21.

33. Fechtner, R.D., Khouri, A.S. Evolving global risk assessment of ocular hypertension to glaucoma. Curr Opin Ophthalmol 2007; 18(2):104-9.

34. Zhao, D., Cho, J., Kim, M.H., Guallar, E. The association of blood pressure and primary open-angle glaucoma: a meta-analysis. Am J Ophthalmol 2014; 158:615-27.

35. Jonas, J., Gusek, G., Naumann, G. Optic disc, cup and neuroretinal rim size, configuration and correlations in normal eyes. Invest Ophthalmol Vis Sci juillet 1988; 29(7):1151-8.

36. Harizman, N., Oliveira, C., Liebmann, J., et coll. The ISNT rule and differentiation of normal from glaucomatous eyes. Arch Ophthalmol (Chicago, Ill.: 1960). Novembre 2006; 124(11):1579-83.

37. Choudhari N., Neog A., Fudnawala V., George R. Cupped disc with normal intraocular pressure: the long road to avoid misdiagnosis. Indian J Ophthalmol novembre 2011; 59(6):491-7.

38. Greenfield D., Siatkowski R., Glaser J., Schatz N., Parrish R. The cupped disc. Who needs neuroimaging? Ophthalmology, octobre 1998; 105(10):1866-74.

39. Greenfield D. Glaucomatous versus nonglaucomatous optic disc cupping: clinical differentiation. Semin Ophthalmol juin 1999; 14(2):95-108.

40. Zeyen T., Miglior S., Pfeiffer N. et coll. Reproducibility of evaluation of optic disc change for glaucoma with stereo optic disc photographs. Ophthalmology 2003; 110(2):340-4. 
41. Weinreb R., World Glaucoma A. Progression of Glaucoma: The $8^{\text {th }}$ Consensus Report of the World Glaucoma Association. Amsterdam: Kugler Publications; 2011, 91-9.

42. Banegas, S., Antón, A., Morilla-Grasa, A., Bogado, M., Ayala, E., Moreno-Montañes, J. Agreement among spectral-domain optical coherence tomography, standard automated perimetry, and stereo photography in the detection of glaucoma progression. Invest Ophthalmol Vis Sci 27 janvier 2015; 56(2):1253-60.

43. Sehi M., Bhardwaj N., Chung Y.S., Greenfield D.S.; Advanced Imaging for Glaucoma Study Group. Evaluation of baseline structural factors for predicting glaucomatous visual-field progression using optical coherence tomography, scanning laser polarimetry and confocal scanning laser ophthalmoscopy. Eye (Lond). déc. 2012, 26(12):1527-3.

44. Sommer A., Miller N.R., Pollack I., Maumenee A.E., George T. The nerve fiber layer in the diagnosis of glaucoma. Arch Ophthalmol déc. 1977; 95(12):2149-56.

45. Kuang T., Zhang C., Zangwill L., Weinreb R., Medeiros F. Article original: Estimating lead time gained by optical coherence tomography in detecting glaucoma before development of visual field defects. Ophthalmology $1^{\text {er }}$ octobre 2015; 122:2002-9.

46. Kerrigan-Baumrind L.A., Quigley H.A., Pease M.E. et coll. Number of ganglion cells in glaucoma eyes compared with threshold visual field tests in the same persons. Invest Ophthalmol Vis Sci 2000; 41(3):741-8.

47. Liu S., Lam S,. Weinreb R.N. et coll. Comparison of standard automated perimetry, frequency-doubling technology perimetry, and short-wavelength automated perimetry for detection of glaucoma. Invest Ophthalmol Vis Sci 2011; 52(10):7325-31.
48. De Moraes C.G., Sehi M., Greenfield D.S. et coll. A validated risk calculator to assess risk and rate of visual field progression in treated glaucoma patients. Invest Ophthalmol Vis Sci 2012; 53(6):2702-7.

49. Leske M.C., Heijl A., Hussein M. et coll. Factors for glaucoma progression and the effect of treatment: the early manifest glaucoma trial. Arch Ophthalmol 2003; 121(1):48-56.

50. Musch D.C., Lichter P.R., Guire K.E. et coll. The Collaborative Initial Glaucoma Treatment Study: study design, methods, and baseline characteristics of enrolled patients. Ophthalmology 1999; 106(4):653-62.

51. Kass, M.A., Heuer, D.K., Higginbotham, E.J. et coll. The Ocular Hypertension Treatment Study: a randomized trial determines that topical ocular hypotensive medication delays or prevents the onset of primary open-angle glaucoma. Arch Ophthalmol 2002; 120(6):701-13; discussion 829-30.

52. Ederer F., Gaasterland D.A., Dally L.G. et coll. The Advanced Glaucoma Intervention Study (AGIS): 13. Comparison of treatment outcomes within race: 10-year results. Ophthalmology 2004; 111(4):651-64.

53. Collaborative Normal-Tension Glaucoma Study Group. The effectiveness of intraocular pressure reduction in the treatment of normal-tension glaucoma. Am J Ophthalmol 1998; 126(4):498-505.

54. Jampel H.D. Target pressure in glaucoma therapy. J Glaucoma 1997; 6(2):133-8.

55. The AGIS Investigators. The Advanced Glaucoma Intervention Study (AGIS): 7. The relationship between control of intraocular pressure and visual field deterioration. Am J Ophthalmol 2000; 130(4):429-40. 